\title{
INJECTIVE COMPACT DISTRIBUTIVE LATTICES ${ }^{1}$
}

\author{
TAE HO CHOE
}

\begin{abstract}
Abstracr. In the category of zero-dimensional compact distributive lattices and their continuous lattice-homomorphisms, it is firstly shown that the injective objects are precisely the compact Boolean algebras, and each object has an injective envelope. Secondly, it is shown that the category of compact distributive lattices and their continuous lattice-homomorphisms has no nontrivial injective object.
\end{abstract}

By a topological lattice, we mean a lattice together with a Hausdorff topology under which the two lattice operations are continuous.

Let $D\left(D_{0}\right)$ be the category of all compact (zero-dimensional respectively) distributive lattices and their continuous lattice-homomorphisms.

All categorical terminologies and notations used in this paper are the same as in [10]. In $D$ (or $D_{0}$ ) it is easy to see that the monomorphisms are precisely morphisms that are one-to-one. As the above abstract, we shall show that each object in $D_{0}$ has an injective extension not containing any properly smaller such extension. Thus the behaviour of essential extensions in $D_{0}$ is quite similar with those of categories of all left modules over a ring [8], and of all partially ordered sets (or all distributive lattices) [2], [3]. The essential extension of $D$, however, behaves very badly.

We shall use $A \wedge B$ to denote the set $\{a \wedge b \mid a \in A$ and $b \in B\}$ for two subsets $A$ and $B$ of a lattice, and dually. $A^{*}$ is the closure of $A$.

1. Injective lattices and injective envelopes in $D_{0}$. A topological lattice is said to have small lattices iff it has a topological basis consisting of open sublattices [6].

LEMMA 1. Every zero-dimensional compact distributive lattice has small lattices consisting of open, convex sublattices.

Proof. Such a lattice $L$ is always topologically and algebraically embedded into a compact Boolean lattice of type $2^{\alpha}[11]$. Therefore $L$ has

Received by the editors February 15, 1972.

AMS (MOS) subject classifications (1970). Primary 54F05; Secondary 18D35.

Key words and phrases. Topological lattice, category, injective, injective envelope, power set topological lattice.

1 This research was supported by the NRC under grant A4809.

(c) American Mathematical Society 1973 
enough characters to separate points [7]. It follows from (Theorems 4 and 5 in [7]) that $L$ has a basis of open neighborhood which are convex sublattices.

LEMMA 2. Every zero-dimensional compact distributive lattice has small lattices consisting of closed, open (=clopen), convex sublattices.

Proof. Let $L$ be such a lattice. It is well known [1] that the operation of forming $C(V)=(L \wedge V) \cap(L \vee V)$ from a subset $V$ of $L$ preserves separately each of the properties of being open, being closed (compact), being a sublattice, and further that $C(V)$ is the smallest convex subset containing $V$. For an open neighborhood $U$ of $a \in L$, we can find an open convex sublattice $W$ of $L$ such that $a \in W \subset U$, by Lemma 1. By hypothesis, there exists a clopen neighborhood $V$ of $a$ such that $V \subset W$. Then $C(V)$ is clopen convex and $C(V) \subset W$. Now let $\Phi$ be the family of all convex open sublattices $V_{\alpha}$ containing $a$ such that $V_{\alpha} \subset C(V)$. Clearly $\Phi$ is not empty by Lemma 1 , and $\Phi$ is inductive under inclusion. Therefore we can find a maximal member $V_{0}$ in $\Phi$. Now we show that $V_{0}$ is closed. Indeed, $V_{0}^{*} \subset$ $C(V)$. Let $p=\operatorname{Inf} V_{0}^{*}$ and $q=\operatorname{Sup} V_{0}^{*}$. Then $p, q \in V_{0}^{*}$. Again choose two open sublattices $V_{1}$ and $V_{2}$ such that $p \in V_{1} \subset C(V)$ and $q \in V_{2} \subset C(V)$. Let $W_{0}=\left(L \vee V_{1}\right) \cap\left(L \wedge V_{2}\right)$. Then $W_{0}$ is an open convex sublattice, and $V_{0}=$ $W_{0}$. Therefore $V_{0}^{*} \subset[p, q] \subset W_{0}=V_{0}$. The proof is complete.

LEMMA 3. In the category $D_{0}$, the Boolean lattice $2(=\{0,1\})$ with the discrete topology is an injective lattice.

Proof. Suppose that $j: A \rightarrow L$ is a monomorphism, and $f: A \rightarrow 2$ is a morphism in $D_{0}$. Let $I=j\left(f^{-1}(0)\right)$ and $J=j\left(f^{-1}(1)\right)$. We assume that $I \neq \varnothing \neq J$, otherwise, the case is trivial. Then $I$ and $J$ are compact sublattices in $L$. Let $a=\operatorname{Sup} I$ and $b=\operatorname{Inf} J$. Since $a \in L \backslash(L \vee J)$, there exists a clopen sublattice $M$ such that $a \in M \subset L \backslash(L \vee J)$. It follows that the clopen ideal $L \wedge M$ contains $L \wedge I$. Similarly, we have a clopen sublattice $N$ such that $b \in N \subset L \backslash(L \wedge M)$, and $L \vee N$ contains $L \vee J$. Clearly, $(L \wedge M) \cap(L \vee N)=$ $\varnothing$. Now consider the family $\Phi$ of all open ideals $V_{\alpha}$ of $L$ such that $L \wedge M \subset$ $V_{\alpha}$ and $V_{\alpha} \cap(L \vee N)=\varnothing$. Then $\Phi$ is not empty, and it is inductive under inclusion. Let $V$ be a maximal member in $\Phi$. Show again that $V$ is closed. In fact, clearly, $V^{*} \cap(L \vee N)=\varnothing$. Setting $x=\operatorname{Sup} V^{*}$, we choose a clopen sublattice $W$ such that $x \in W \subset L \backslash(L \vee N)$. $L \wedge W$ is an open ideal of $L$ containing $L \wedge M$, and $(L \wedge W) \cap(L \vee N)=\varnothing$. Hence we have $V=L \wedge W$ by the maximality of $V$. Thus $x \in V$, and hence $V=V^{*}$. Since $V$ is an ideal, we have $V=x \wedge L$. Further, $x$ is a meet-irreducible element. For, if $x=\alpha \wedge \beta$, then either $\alpha \notin L \vee N$ or $\beta \notin L \vee N$. Say $\alpha \notin L \vee N$. Again choose a clopen sublattice $W$ such that $\alpha \in W$ and $W \cap(L \vee N)=\varnothing$. Then $V \subset W \wedge L$, and hence $V=W \wedge L$. Thus $\alpha=x$. 
Now we define $g: L \rightarrow 2$ as follows: $g(y)=0$ if $y \in V$ and $g(y)=1$ if $y \notin V$. Then it is easy to see that $g$ is a continuous lattice-homomorphism which is an extension of $f$, i.e., $f=g \circ j$.

By a power set (topological) lattice, we mean a product topological lattice $2^{\alpha}$ of $\alpha$ lattices 2 with the discrete topology. It is known [5] that every compact Boolean topological lattice is iseomorphic (algebraically and topologically) with a power set topological lattice.

THEOREM 1. In the category $D_{0}, Q$ is injective iff $Q$ is a power set lattice.

Proof. Suppose that $Q$ is injective. By [11], $Q$ is embedded into a power set lattice $2^{x}$ algebraically and topologically. For the identity map: $Q \rightarrow Q$, there exists a continuous lattice-homomorphism: $2^{\alpha} \rightarrow Q$, which is onto. Thus $Q$ is a retract of $2^{\alpha}$. Hence $Q$ is also a power set lattice. The converse is trivial by Lemma 3 .

The assertion ' $2^{\alpha} \leqq 2^{\beta}$ implies $\alpha \leqq \beta$ for any cardinals $\alpha, \beta$ " is known to be independent as an axiom of set theory. However, the following lemma is easy to prove:

Lemma 4. For tho cardinals $\alpha, \beta$, if the power set lattice $2^{\alpha}$ is latticeembedded into $2^{\beta}$, then $\alpha \leqq \beta$.

Proof. Let $f$ be a one-to-one lattice-homomorphism of $2^{\alpha}$ into $2^{\beta}$. For each $x \in \alpha, f$ carries the characteristic function of $\{x\}$ to the characteristic function of a subset $S_{x}$ of $\beta$. Let $S_{\varnothing}$ be the subset of $\beta$ whose characteristic function is the image of 0 (=the characteristic function of $\varnothing)$ in $2^{\alpha}$ under $f$. Then, clearly, $S_{x} \mid S_{\varnothing} \neq \varnothing$ for all $x \in \alpha$, and $\left(S_{x} \mid S_{\varnothing}\right) \cap\left(S_{y} \mid S_{\varnothing}\right)=\varnothing$ for all $x$ and $y \in \alpha(x \neq y)$. Let $\beta_{0}$ be the union of $S_{x} \mid S_{\varnothing}(x \in \alpha)$. Then $\beta_{0} \geqq \alpha$, thus $\beta \geqq \alpha$.

For $L \in D_{0}$, a power set topological lattice $2^{\alpha}$ is said to be generated by $L$ if $\alpha$ is the first cardinal among all those $\alpha$ such that $L$ is embedded into $2^{\alpha}$ algebraically and topologically. It is then easy to see that the embedding preserves the least and the greatest elements.

THEOREM 2. Every $L \in D_{0}$ has an injective envelope that is the power lattice generated by $L$.

Proof. Let $2^{x}$ be the power lattice generated by $L$, and $i$ be the embedding of $L$ into $2^{\alpha}$. Let [iL] be the Boolean algebra algebraically generated by the underlying lattice of $i(L)$ in $2^{\alpha}$. Then $[i L]^{*}$ is a compact Boolean topological lattice. Thus it is a power set lattice $2^{\beta}$, in which $L$ is algebraically and topologically embedded. By Lemma $4, \beta \leqq \alpha$. Thus $\alpha=\beta$. Hence $[i L]$ is a dense subset of $2^{\alpha}$. Now consider the following:

$$
L \stackrel{i}{\longrightarrow}[i L] \subset 2^{\alpha} \stackrel{g}{\rightarrow} M,
$$


where $i$ is the above embedding, and $g$ is a morphism: $2^{\alpha} \rightarrow M$ such that $g \circ i$ is a monomorphism.

Let $g\left(2^{\alpha}\right)=2^{\gamma}$. Since $2^{\alpha}$ is injective in $D_{0}$, for the monomorphism $g \circ i$ and $i$, there exists a morphism $F: 2^{\gamma} \rightarrow 2^{\alpha}$ such that $F \circ(g \circ i)=i$. Hence $F \circ g$ is the identity on $i(L)$. Since $g: 2^{\alpha} \rightarrow 2^{\gamma}$ and $i$ preserve the least and the greatest elements, $F \circ g$ preserves the complement operations of [iL]. It follows that $F \circ g$ is the identity on $[i L]$, and hence it is the identity on $2^{\alpha}$. Thus $g$ is a monomorphism. The proof is complete.

2. Injective lattices in $D$. In this section we shall show that every injective lattice in $D$ is trivial, namely the only injective lattice is a singleton lattice. Suppose that $Q$ is injective in $D$. If the cardinal of $Q$ is greater than 2 , then for $x \in Q(0 \neq x \neq 1)$ let $M$ be the sublattice $\{0, x, 1\}$ of $Q$, and let $N$ be a discrete Boolean algebra $\left\{0, x, x^{\prime}, 1\right\}$. For the inclusion $i$ of $M$ into $N$, we can find a morphism $f: N \rightarrow Q$ such that $f \circ i=j$, where $j$ is the inclusion of $M$ into $Q$. Clearly $f\left(x^{\prime}\right)$ is a complement element of $f(x)(=x)$ in $Q$. Therefore $Q$ must be a compact Boolean lattice.

From this observation, it is easy to see the following:

THEOREM 3. Every injective lattice in $D$ is trivial.

Proof. Let $Q$ be injective. Then $Q$ is a power set lattice, say $2^{x}$. Suppose that $\alpha \geqq 1$. Let $j$ be the monomorphism: $2 \rightarrow I$, where $I=[0,1]$ is the unit interval with the ordinary topology and order, such that $j(0)=0$ and $j(1)=1$. For the morphism $i: 2 \rightarrow 2^{x}$ with $i(0)=0$ and $i(1)=1$, there exists a morphism $f: I \rightarrow 2^{\alpha}$ such that $f \circ j=i$. But $2^{\alpha}$ is a totally disconnected nondegenerate space. This is a contradiction.

\section{REFERENCES}

1. L. W. Anderson, One dimensional topological lattices, Proc. Amer. Math. Soc. 10 (1959), 715-720. MR 21 \#6401.

2. B. Banaschewski and G. Bruns, Injective hulls in the category of distributive lattices, J. Reine Angew. Math. 232 (1968), 102-109. MR 38 \#5888.

3. Categorical characterization of the MacNeille completion, Arch. Math. (Basel) 18 (1967), 369-377. MR 36 \#5036.

4. R. Balbes, Projective and injective distributive lattices, Notices Amer. Math. Soc. 13 (1966), 740. Abstract \#66T-492.

5. T. H. Choe, On compact topological lattices of finite dimension, Trans. Amer. Math. Soc. 140 (1969), 223-237. MR 39 \#1366.

6. Locally compact lattices with small lattices, Michigan Math. J. 18 (1971), 81-85.

7. E. B. Davies, The existence of characters on topological lattices, J. London Math. Soc. 43 (1968), 217-220. MR 37 \#112.

8. B. Eckmann and A. Schopf, Über injektive Modulen, Arch. Math. 4(1953), 75-78. MR 15, 5. 
9. P. R. Halmos, Injective and projective Boolean algebras, Proc. Sympos. Pure Math., vol. 2, Amer. Math. Soc., Providence, R.I., 1961, pp. 114-122. MR 25 \#1121.

10. B. Mitchell, Theory of categories, Pure and Appl. Math., vol. 17, Academic Press, New York, 1965. MR 34 \#2647.

11. K. Numakura, Theorems on compact totally disconnected semigroups and lattices, Proc. Amer. Math. Soc. 8 (1957), 623-626. MR 19, 290.

Department of Mathematics, McMaster University, Hamilton, Ontario, CANADA 